Meta

Journal des traducteurs

Translators' Journal

\title{
Les mésaventures du merle : les américanismes chez Emily Dickinson
}

\section{Charlotte Melançon}

Volume 45, numéro 1, avril 2000

La traduction littéraire au Canada

Literary Translation in Canada

URI : https://id.erudit.org/iderudit/003616ar

DOI : https://doi.org/10.7202/003616ar

Aller au sommaire du numéro

Éditeur(s)

Les Presses de l'Université de Montréal

ISSN

0026-0452 (imprimé)

1492-1421 (numérique)

Découvrir la revue

Citer cet article

Melançon, C. (2000). Les mésaventures du merle : les américanismes chez

Emily Dickinson. Meta, 45(1), 80-90. https://doi.org/10.7202/003616ar
Résumé de l'article

La proximité géographique ou culturelle ne confère aucune compétence pour traduire un auteur. L'œuvre offre toujours une résistance que la reconnaissance immédiate et quasi instinctive de certaines réalités physiques, par exemple, ne saurait vaincre. Si l'œuvre appartient à un lieu donné, elle n'en dépend évidemment pas. Cela dit, pourquoi la méconnaissance de certains faits de civilisation et de réalités géographiques, de la faune et de la flore, entraîne-t-elle tant d'inexactitudes, voire de contresens, dans les traductions françaises d'Emily Dickinson?
Ce document est protégé par la loi sur le droit d'auteur. L'utilisation des services d'Érudit (y compris la reproduction) est assujettie à sa politique d'utilisation que vous pouvez consulter en ligne.

https://apropos.erudit.org/fr/usagers/politique-dutilisation/ 


\title{
Les mésaventures du merle: les américanismes chez Emily Dickinson
}

\author{
CHARLOTTE MELANÇON \\ Traductrice et écrivain
}

\begin{abstract}
RÉSUMÉ
La proximité géographique ou culturelle ne confère aucune compétence pour traduire un auteur. L'œuvre offre toujours une résistance que la reconnaissance immédiate et quasi instinctive de certaines réalités physiques, par exemple, ne saurait vaincre. Si l'œuvre appartient à un lieu donné, elle n'en dépend évidemment pas. Cela dit, pourquoi la méconnaissance de certains faits de civilisation et de réalités géographiques, de la faune et de la flore, entraîne-t-elle tant d'inexactitudes, voire de contresens, dans les traductions françaises d'Emily Dickinson?
\end{abstract}

\section{ABSTRACT}

Geographic or cultural proximity is not an absolute prerequisite for translating the work of an author. The work always offers some resistance which immediate and instinctive recognition of related physical realities cannot readily overcome. Although the setting is a given environment, this is not of the essence. That being said, one wonders why misunderstanding of certain cultural details and geographical facts has resulted in so many inaccuracies and mistranslations in the French translations of Emily Dickinson.

\section{MOTS-CLÉS/KEYWORDS}

traduction littéraire, ornithologie, Emily Dickinson, lexique particulier à l'Amérique du Nord

[...] each Mind is itself, like a distinct Bird (L. 457)

Each Consciousness must emigrate

And lose its neighbor once (P. 911)

Il ne faut pas confondre l'intérêt qu'Emily Dickinson a porté aux choses de la nature avec les loisirs des jeunes filles de bonne famille. Cette passion tient en fait de l'esprit des Lumières, ce qui peut paraître étonnant à l'époque romantique où elle a vécu. Et pourtant, nombre d'attitudes et de pratiques la rattachent à la volonté de savoir encyclopédique du XVIII ${ }^{\mathrm{e}}$ siècle qui, sous l'impulsion d'Emerson, se renouvelait alors en Nouvelle-Angleterre. On a dit de sa conférence «The American Scholar», prononcée à Harvard en août 1837, qu'elle était la déclaration d'indépendance intellectuelle des États-Unis. C'est qu'elle s'adressait, à son tour, au common man, appelé au savoir autant qu'à la liberté politique; Emily Dickinson a entendu cet appel.

Deux des poèmes qu'elle consacre à l'oiseau-mouche (P. 334 et 1463), par exemple, reprennent les images mêmes de Buffon dans l'Histoire naturelle (Morency 1992 : 145); on sait par ailleurs que, sa vie durant, elle échangera bulbes et semences avec ses correspondants comme un Haendel et un Telemann, qu'après la construction du conservatory, elle s'adonnera à la culture des fleurs exotiques — notamment la jacinthe et le jasmin du Cap — et qu'elle tentera de domestiquer diverses plantes indigènes 
comme le lis rouge. Quant à son jardin, rappelons qu'il ne s'agit pas de deux ou trois plates-bandes ornementales, mais d'une demie acre de terre, ce qui, d'évidence, est considérable. On connaît malheureusement peu les relations qu'elle a eues (ou pu avoir) avec Frederick Olmstead, l'architecte-paysagiste qui conçut à la même époque presque tous les parcs et jardins d'Amérique, notamment Central Park, South Park et le Mont-Royal. Mais, chose sûre, si le Homestead et les Evergreens représentaient pour lui un modèle de propriété rurale, il va de soi qu'Emily y était pour beaucoup (Brooks 1940: 317). Bref, ce travail impliquait des connaissances, une application minutieuse et régulière qui s'opposent tout à fait à l'image victorienne de la nonne rêvant au milieu de ses lis blancs et que plusieurs critiques ont tenté de rattacher à la peinture préraphaélite. Que non! Dickinson se salissait les mains.

Bien que recluse pendant plus de la moitié de sa vie, elle avait acquis, au cours de son enfance et de son adolescence, de solides connaissances sur le terrain (champs, sous-bois, forêts, montagnes) comme en témoignent les lettres qui nous restent de cette époque, ainsi que son herbier avec ses noms latins dûment imprimés. Amherst College, en outre, était réputé pour son enseignement des sciences naturelles, et elle y suivit les leçons du professeur Edward Hitchcock, auteur reconnu pour ses ouvrages de géologie et de botanique (Sewall 1974:342-347). Loin d'affaiblir sa perception du monde, c'est comme si son retrait de la vie civile l'avait au contraire avivée, comme si son esprit d'observation s'en était trouvé aiguisé: Dickinson transformera dès lors les carreaux de ses fenêtres "en télescopes ou en microscopes », selon l'image emersonnienne, et développera une acuité auditive remarquable.

Dans une lettre de 1863 à Higginson, elle écrit que «le surnaturel n' [est] que le naturel, révélé» (L. 281). Il importe de prendre en considération autant le second terme que le premier puisque la nature constitue effectivement le lieu à partir duquel s'accomplit la transcendance, où se révèle le ravissement poétique: la vision du monde s'inscrit toujours chez elle dans une physique des choses, même si la connaissance expérimentale ne constitue pas une fin en soi $^{2}$.

Cela exige du traducteur un redoublement de vigilance, car seul le souci de nommer correctement ce monde physique qui l'a ravie permet de le comprendre et, par conséquent, de le traduire. Souvent leurrés par des dictionnaires qui ne tiennent pas compte des réalités américaines, les traducteurs européens sont parfois amenés à dénaturer la réalité physique dans laquelle la poésie de Dickinson prend racine. Depuis nombre d'années, à mesure que je lis les traductions de ses poèmes et de sa correspondance, je ne cesse d'être frappée par l'incertitude de ces traducteurs face à la faune et à la flore nord-américaines. La traduction du fameux Indian pipe (P. 1250; il s'agit du Monotropa uniflora) par calumet l'illustre exemplairement: tout se passe comme si la réalité même de la fleur avait été complètement sacrifiée à une sorte de poncif folklorique. Or il est impossible de comprendre le poème sans une identification juste de cette plante très particulière (américaine et asiatique) et qui s'appelle, il est vrai, fort prosaïquement, monotrope $e^{3}$.

Mais puisque l'objet de cet article est l'ornithologie et que la matière est déjà trop vaste, je ne ferai allusion à la botanique que dans les rapports qu'elle entretient avec celle-ci. Le Bobolink me servira de point de départ. Cet oiseau est traduit indifférem- 
ment par babillard, bruant, geai, loriot, merle ou perruche, toutes appellations erronées, qui trahissent un embarras certain. Fait plus étonnant encore, on retrouve chez un même traducteur des traductions différentes pour ce même oiseau, comme s'il s'agissait d'un objet interchangeable qui ne possèderait pas d'identité propre ${ }^{4}$. La traduction de Bobolink - goglu — se trouve pourtant dans le Harrap's et dans tous les ouvrages scientifiques consacrés à l'ornithologie de l'Amérique du Nord ${ }^{5}$.

Il s'agit d'un nom populaire qui s'est imposé au Québec, supplantant celui d'Ortolan de riz qu'on cherchait encore à lui donner au début du siècle et qui est tout à fait impropre. Le mot «goglu», déformation de «goguelu», est un vieux mot dialectal qui signifie dans certaines régions de France: fanfaron, présomptueux, ou bien: plaisantin, hâbleur. Ces diverses significations ont sans doute été renforcées par le chant lui-même, plus précisément par les deux premières syllabes glougloutantes, sorte de guing-gong, avec lesquelles le Goglu amorce sa ligne musicale. Son nom anglais est Bobolink. Étrangement, c'est sous ce vocable que le connaissent les ornithologistes de France. (Morency 1992: 118-119)

Il ne s'agit sûrement pas de tirer la couverture à soi et de prôner les canadianismes ou les québécismes. Mais il a bien fallu que le Canada français nomme cet oiseau d'Amérique qui couvre le tiers environ de son territoire ${ }^{7}$ Étant donné, par ailleurs, la place qu'il occupe dans le bestiaire de Dickinson, il est essentiel de le reconnaître pour ce qu'il est; sans compter ses occurrences dans la correspondance, le goglu est l'oiseau qui figure le plus fréquemment dans les poèmes, juste après le merle, et toutes les métaphores qui s'y rapportent sont d'une parfaite exactitude. Petit comme un moineau mais doté d'un dispositif sonore unique, chantant en plein vol avec une truculence inouïe, le goglu offre l'image même de la jubilation et de la transgression, combinant l'audace du fanfaron et la modestie du franciscain, si caractéristiques d'ailleurs de la poésie dickinsonnienne. Dans le poème 1279, Dickinson en fait un hérétique et un joyeux sorcier, sorte de Puck du Nouveau Monde; dans un autre, le goglu envoie carrément "valser le Décalogue»(P. 1591). Mais déjà dans l'un des premiers poèmes, cet oiseau était associé à son renoncement à toute religion instituée:

Some keep the Sabbath going to ChurchI keep it, staying at Home -

With a Bobolink for a Chorister -

And an Orchard, for a Dome -

Some keep the Sabbath in Surplice -

I just wear my Wings -

And instead of tolling the Bell, for Church,

Our little sexton - sings.

God preaches, a noted Clergyman -

And the sermon is never long,

So instead of getting to Heaven, at last-

I'm going, all along. (P. 324)

Certains vont à l'Église le Dimanche -

Moi, je reste à la Maison -

Le Goglu est mon Chantre -

Et le Verger, ma Voûte -

Certains portent un Surplis le Dimanche -

Moi, je n'ai que mes Ailes - 
Et au lieu de sonner la Cloche,

Notre petit Sacristain chante.

Dieu prêche, c'est un fameux Ministre -

Et le sermon n'est jamais long.

Ainsi, au lieu d'aller au Ciel -

Je suis, tout compte fait, ma route.

La petite taille du goglu et la tonalité pétillante de son chant ne peuvent s'appliquer au merle et encore moins à la perruche, oiseau de volière au grincement étouffé. Rien ne saurait être plus étranger à l'esprit de Dickinson que cet oiseau gardé en cage. Tout le poème dit le contraire: l'indépendance du poète à l'égard de l'Église, l'affirmation du sacré à l'intérieur de la nature proche; le goglu est un païen, un Papageno.

Mais c'est sur le Robin que les traducteurs européens achoppent le plus souvent. C'est pourtant l'oiseau dickinsonien par excellence, celui auquel elle ne cesse de se référer, auquel elle s'est très tôt identifiée: le merle d'Amérique, Turdus migratorius (Turdidoe). Il est répertorié dans tous les dictionnaires d'usage, le Webster's, ${ }^{8}$ l'Oxford English Dictionary, le Random House et dans le dictionnaire bilingue Harrap's. Les dictionnaires d'usage français, comme le Robert, par exemple, n'en font pas état, et c'est sans doute ce qui a créé toute cette confusion. Le merle qu'on y décrit est évidemment le merle européen : "Oiseau passériforme (Passereaux-Turdidés), dentirostre, au plumage sombre, sans tache (ce qui le différencie de la grive)».

N'y reconnaissant pas, à juste titre, le merle américain, les traducteurs européens ont fait appel ici encore à différents vocables: grive, passereau, Pierrot, pinson, rouge-gorge. On utilise le plus fréquemment rouge-gorge, réflexe inévitable, semblet-il, puisqu'il reprend le terme même auquel ont eu recours les premiers Français débarquant en Amérique:

Les premiers colons l'ont appelé Rouge-gorge, mais à tort, car ils croyaient retrouver le fameux Rouge-gorge de l'Ancien Monde. Cependant, notre Merle est un oiseau aux dimensions beaucoup plus imposantes, et sauf pour la coloration de sa poitrine, il ne ressemble en rien au Rouge-gorge de l'Ancien Monde. (Godfrey 1967: 340)

L'American Robin, par ailleurs, est l'une des rares espèces qui a pu bénéficier de la colonisation, le merle n'aimant pas les bois denses et profonds; son aire de dispersion, très vaste, s'étend désormais à presque tout le continent. Son caractère familier fait qu'on l'appelle, ici, en Amérique du Nord, communément et tout simplement Robin ou merle: cela porte plus à conséquence en français qu'en anglais puisque la famille des Turdidés regroupe un grand nombre de sous-espèces qui portent également le nom de merles: le merle bleu à poitrine rouge (Bluebird $\left.{ }^{9}\right)$, le merle bleu de l'Ouest, le merle bleu azuré, le merle à dos roux et le merle fauve. Mais on n'appelle communément Robin que l'American Robin - les autres membres de la famille portant tous des noms différents. Comme Dickinson l'écrit délicieusement:

They registred in Nature's Book

As Robins - Sire and Son - (P. 1570, v. 5-6)

Ils sont inscrits au Livre de Nature

Sous le nom de Merles - Père et Fils - 
En quoi de telles précisions peuvent-elles affecter la traduction? Voyons la première strophe du poème 182 , l'un des plus fréquemment traduits:

If I shouldn't be alive

When the Robins come,

Give the one in Red Cravat,

A Memorial crumb.

$\mathrm{Si}$ au retour des rouges-gorges

Je n'étais plus en vie,

$\mathrm{Au}$ cravaté de rouge donne

La miette commémorative. (Bosquet 1957)

Si je n'étais plus là

Quand les Rouges-gorges viendront,

Donnez à celui à la Rouge Cravate,

La miette du Souvenir. (Denis 1988)

Si je n'étais plus en vie

Quand viendront les Grives,

Donne au Cravaté de Rouge,

Une miette Commémorative. (Malroux 1989)

Si je n'étais plus en vie

Quand les Passereaux s'en viennent,

Donne au Cravaté de Rouge

Une miette - Mémoriale. (Leyris 1995)

Quand viendront les Rouges-gorges

$\mathrm{Si}$ je ne suis plus en vie

Donne au Cravaté de Rouge

Une miette Commémorative. (Malroux 1998)

Si je ne suis plus vivante

Au retour des Rouges-Gorges,

Donne à l'Étranglé de Rouge,

Une miette au Mémorial. (Reumaux 1998)

Aucune de ces traductions ne reprend le nom de merle; rouge-gorge revient quatre fois, grive et passereau une fois chacun, ces deux derniers évitant heureusement le redoublement pléonastique de «rouge» aux deuxième et troisième vers. La traduction qui s'en approche le plus est celle de Pierre Leyris puisque le merle appartient à la famille des passereaux; mais comme celle-ci comprend également les grives, cette traduction, trop vague, ne peut être maintenue. Je propose la version suivante:

Si je ne suis plus en vie

Quand reviendront les Merles,

Au Cravaté de Rouge donne

En Souvenir un peu de mie.

La cravate rouge suffit à identifier le merle d'Amérique; mais dans une édition européenne, il faudrait sans doute préciser dans une note de quel oiseau il s'agit. Quant à la traduction de Philippe Reumaux, son «Étranglé de Rouge » est un contresens puisque la Red Cravat sert, d'une part, à décrire et à identifier l'oiseau et qu'elle renvoie, d'autre part, à toute une série d'images qui, chez Dickinson, expriment la 
bonté, la tendresse et la reconnaissance ${ }^{10}$; très tôt, d'ailleurs, elle se définira ellemême comme l'un d'eux: Moi - avec ma Gorge rouge - / Et mes Rimes - (P. 250).

Comme le merle, d'autre part, signale l'arrivée du printemps en NouvelleAngleterre, Dickinson associe souvent la couleur rouge au mois de mars. Lorsqu'elle écrit, par exemple, j'ai le cour [...] pourpre comme Mars (L. 315), elle renvoie bien sûr au Pèlerin rouge (P. 773), et au retour à la vie qu'il symbolise.

L'affection qu'elle éprouve pour cet oiseau commun et familier tient avant tout à son appartenance géographique:

The Robin's my Criterion for Tune -

Because I grow - where Robins do -

But, were I Cuckoo born -

I'd swear by him -

[...]

I see - New Englandly -

[...]

Provincially - (P. 285, v. 1-4, 15, 17)

J'accorde mon Chant sur le Merle Car je vis - là où vivent les Merles Je serais née chez le Coucou -

Que je ne jurerais que par lui -

[...]

Je vois - comme on voit les choses

En Nouvelle-Angleterre -

[...]

Provincialement -

Le «Criterion for Tune» du premier vers est capital, puisqu'il définit une norme, et c'est l'une des raisons pour lesquelles il importe tant d'identifier correctement cet oiseau. Cette appellation précise importe pour toutes les occurrences du Robin; à la fois règle et modèle, le merle implique l'idée de constance et de connivence. Ce n'est donc pas un hasard si, dans ce poème où Dickinson dit voir les choses New Englandly, elle a élu le merle ${ }^{11}$.

Ce lieu qui la définit, la Nouvelle-Angleterre, renvoie à toute une culture calviniste; elle appelle non sans raison le Homestead que fréquentent ces passereaux son Jardin Puritain (L. 685). Le merle, en effet, revient à travers tout un réseau de métaphores qui tracent un portrait social et moral de la Nouvelle-Angleterre:

\author{
The Robin is a Gabriel \\ In humble circumstances - \\ His Dress denotes him socially, \\ Of Transport's Working Classes - \\ He has the punctuality \\ Of the New England Farmer - \\ The same oblique integrity, (P. 1483, v. 1-7) \\ Le Merle est un Gabriel \\ De modeste condition - \\ Il appartient - on le voit à sa Robe - \\ À la Classe Ouvrière des Transports -
}


Ponctuel comme un Fermier

De la Nouvelle-Angleterre -

Il en a aussi l'oblique probité -

Le rapprochement du merle-ouvrier et de l'archange modeste (mais archange tout de même) se concrétise dans l'image du fermier. Figure emblématique de la philosophie d'Emerson, le fermier est le producteur libre et efficace, consciencieux et astucieux, bienfaiteur de l'humanité. Ces valeurs se retrouvent chez Dickinson; on ne peut donc pas, comme on le fait souvent, traduire farmer par "paysan » qui a ici des connetations d'Ancien Régime. Reprenant une expression déjà en usage au milieu du XVIII ${ }^{\mathrm{e}}$ siècle, Balzac dit du paysan français qu'il est un «nègre blanc» (Balzac 1949: 210-211).

Aux qualités d'intégrité ${ }^{12}$ et d'application consciencieuse du merle, Dickinson en ajoute une autre - celle de démocrate:

The Robins stand as stick today

As flakes of snow yesterday -

On fence - and Roof - and Twig! (P. 64, v. 13-15)

Les Merles se tiennent en rangs serrés

Comme hier les flocons de neige

Sur la grille - le Toit - les Branches!

J'ignore si Dickinson songe ici au phénomène météorologique du Robin snow neige qui survient après l'arrivée du printemps ${ }^{13}$ — mais cela ne change en rien l'analogie. La «commune neige» (P. 325, 216, 126), en effet, nivelle, abolit les différences:

It makes an Even Face

Of Mountain, and of Plain -

Unbroken Forehead from the East

Unto the East again - (P. 311, v. 5-8)

Elle Égalise le Visage

De la Montagne et de la Plaine -

En lisse le Front

De l'Est jusqu'à l'Est -

C'est leur nombre, leur abondance, qui relie les merles et les flocons de neige. Mais la neige apporte une idée d'uniformité qui consacre leur caractère familier et banal: le merle est le commun des mortels, le citoyen ordinaire, le «Sweet Countryman» (P. 441). Dans le poème 153, lorsque Dieu vient faucher les hommes, c'est du nom de merles que Dickinson les désigne:

Christ robs the Nest -

Robin after Robin

Smuggled to Rest! (v. 14-16)

Le Christ pille le Nid -

Merle après Merle

Passés en fraude au Paradis!

Mais le merle est aussi chez elle une métaphore d'écriture; le poème 1483, cité plus haut, comporte une variante significative: 


\section{The Robin is a Troubadour \\ In humble circumstances - \\ Le Merle est un Troubadour \\ De modeste condition -}

Toutes les occurrences de «troubadour» renvoient à cet oiseau, sauf une qui désigne le roi David. Il est difficile, en pareil cas, de ne pas faire du poète biblique un merle ou de ne pas identifier les merles à de petits psalmistes, d'autant que les quatrains de Dickinson sont modelés sur l'hymn meter du Common Prayers'Book. Qu'elle ait accordé sa préférence aux psaumes, c'est ce que dit ici le merle; le goglu, quant à lui, était plus radical, envoyant même promener les dix commandements. Mais le langage de l'un et l'autre mêle le chant sacré à la poésie profane.

S'il est souvent une image de la jubilation, de l'ivresse du printemps (P. 128, 864, 195), le merle n'en reste pas moins lié à la modération, à la mesure. Il n'y a rien chez lui de la truculence du goglu, rien non plus de l'effacement du troglodyte ou de la frénésie de l'oiseau-mouche. Le merle est à la mesure humaine, du common man: c'est un fermier qui lit les versets de la Bible. Cela explique sans doute l'une des images les plus étonnantes de l'oiseau, dans laquelle il est difficile de ne pas reconnaître l'affection que Dickinson lui portait; elle figure dans une lettre de mars 1884 :

Do you remember writing to us you should «write with the Robins?» They are writing now, their Desk in every passing Tree [...] -

Vous rappelez-vous nous avoir dit que « vous écririez avec les Merles»? Ils sont en train d'écrire, à présent, leur Pupitre dans chaque Arbre où ils passent [...] - (L. 890)

Les merles ne chantent pas seulement, ils écrivent. Dickinson possédait une table portative qu'elle déplaçait avec elle un peu partout dans la maison, en particulier dans sa serre; elle y aurait beaucoup écrit, au témoignage de sa sœur. Mais l'image du merle-scribe joue à un autre niveau. Les oiseaux ayant donné à l'humanité un de ses instruments d'écriture les plus familiers - la plume — c'est comme si Dickinson rendait ici aux merles ses propres outils d'écriture: non seulement son crayon ou sa plume, mais sa table. Cette réciprocité porte en fait une valeur symbolique; le merle incarne le poète par excellence, mais Dickinson aussi est cet oiseau qui chante le chant de renouveau de l'Amérique.

Si Emily Dickinson a pu écrire que chaque Esprit est lui-même, tel un oiseau distinct, cela implique qu'elle a su reconnaître chaque oiseau pour ce qu'il est. La vingtaine d'oiseaux que j'ai pu relever sont presque tous familiers de la côte Est américaine; même si le merle et le goglu dominent nettement, elle cite aussi l'aigle, l'alouette, le cardinal, le carouge, le colibri ou l'oiseau-mouche, la corneille, l'engoulevent, le geai, le moineau, le moucherolle, la perdrix, le pluvier, le vautour, etc. Peu d'oiseaux mythologiques ou bibliques, à part l'alcyon et la colombe que Dickinson appelle soit «dove», soit «columba», cette dernière appellation renvoyant toutefois, comme l'a fait remarquer Northrop Frye (1963: 210), au nom du découvreur. La variété de ce bestiaire appartient à son art poétique, au regard qu'elle pose sur les choses; la flore de la Nouvelle-Angleterre appellerait des remarques analogues. Elle a nommé toutes 
ces choses avec une telle exactitude et une telle imagination - ce n'est pas contradictoire - qu'on peut les reconnaître à coup sûr dans ses poèmes. Plantes et bêtes portent sa signature, mais elles signent aussi son lieu de vie.

Ce qui gêne dans les traductions européennes, souvent fort belles d'ailleurs — la question n'est pas là - c'est de ne pas y retrouver la fibre américaine de sa culture. On ne cesse de revenir sur son enracinement en Nouvelle-Angleterre et pourtant on escamote nombre de ses caractères les plus visibles: comment peut-on traduire dans le poème 285 le vers «The Robin's my Criterion for Tune» si on n'identifie pas le merle d'Amérique? On peut toujours soutenir qu'on traduit davantage des valeurs, ou un esprit, que des mots. Tout n'est certes pas traduisible, mais lorsque des mots, répertoriés dans les dictionnaires, appartiennent à l'usage nord-américain, c'est appauvrir sa traduction de ne pas les utiliser. Les recours quasi systématiques à des équivalents européens brouillent les poèmes - leur rigueur et leur justesse - pour ne rien dire de leur profondeur historique et de leur inscription dans une culture. Certes, les poèmes de Dickinson sont aussi imprégnés de culture européenne et universelle, de Shakespeare et de la Bible notamment, mais sa vision du monde se fonde d'abord sur son lieu de vie. La diversité des termes auxquels ont recours les traducteurs européens pour traduire un seul et même oiseau, par exemple, manifeste de toute évidence une méconnaissance de ce lieu.

Une autre difficulté — qui est étroitement liée à cette méconnaissance — tient à ce que j'appellerais la sur-poétisation des traductions. Cela fait peut-être plus "poétique» de traduire Wren par roitelet, farmer par paysan, country town par bourg de province, suburbs par faubourgs, mile par lieue, corn par blée ${ }^{14}$, etc., mais cela ne rend compte ni des réalités américaines, ni du réalisme parfois si cru des poèmes de Dickinson. Comme Emerson, elle adhère moralement et socialement à la géographie du pays, à son climat, à son système de poids et mesures, bref, à sa nature et à ses institutions particulières. Nommer les réalités américaines, c'était non seulement les reconnaître pour ce qu'elles sont, mais manifester à l'égard de l'Ancien Monde une indépendance fondamentale.

\section{NOTES}

1. Dans cet article, les œuvres d'Emily Dickinson sont désignées par les abréviations L (pour Letters, 1958) et $\mathrm{P}$ (pour Poems, 1955) suivies du numéro du texte.

2. Voir la L. 882 : «I have made a permanent Rainbow by filling a Window with Hyacinths, which Science will be glad to know, and have a Cargo of Carnations, worthy of Ceylon, but Science and Ceylon are Strangers to me, and I would give them both for one look of the gone Eyes, glowing in Paradise [...] ». Cette déclaration n'infirme en rien son attitude à l'égard de la science; même si elle l'associe à un pays aussi exotique que le Ceylan, cette phrase dit que rien ne saurait atténuer le chagrin que la mort récente de tant de proches lui a apporté.

3. Voir la L. 769. L'«orobanche» (Broomrape) s'en rapproche, mais appartient à une autre famille, d'origine européenne (Niering et Olmstead 1979: 665-666).

4. On pourrait faire la même remarque à propos du Phebe (le moucherolle), traduit soit par vanneau, soit par gobe-mouche. Je ne comprends pas non plus pourquoi on traduit Wren (troglodyte) par roitelet (Kinglet) ou Crow (corneille) par corbeau; il s'agit peut-être, dans ce dernier cas, de l'attraction du Raven de Poe.

5. L'ouvrage de référence le plus commode et le plus sûr est le Guide d'identification des oiseaux de l'Amérique du Nord, National Geographic Society, traduction française aux éditions Broquet, Montréal (Scott 1988).

6. Tout le chapitre intitulé Un ménestrel explosif est consacré à cet oiseau; sur l'étymologie de Bobolink [Bob'o' Lincoln] (L. 223), voir p. 124. «Goguelu» ne se trouve ni dans le Robert ni dans le Littré; 
ce dernier, toutefois, cite «gogue»: "Terme vieilli. Plaisanterie, divertissement.» Il nous est resté dans «goguenard» et dérivés. Je trouve fort astucieux que certains traducteurs italiens, ne trouvant évidemment pas d'équivalent dans leur langue, aient traduit Bobolink par bobolinco; d'autres ont conservé le terme américain.

7. Le bobolink change de nom en anglais au fil de ses migrations vers le sud: on l'appelle ricebird en Caroline du Sud et butterbird en Jamaïque. Mais son nom le plus répandu, sinon unique, en Nouvelle-Angleterre est bobolink.

8. Le Webster's qu'utilisait Dickinson distingue le merle américain du merle européen et du merle asiatique.

9. On traduit parfois Bluebird par gorge-bleue; ce qu'on appelle en Amérique le gorge-bleue (Bluethroat) ne vit que sur la côte du Pacifique. Qui plus est, le merle bleu n'a pas la poitrine bleue, mais rousse; ce sont les plumes du dos, de la tête et de la queue qui le sont.

10. Voici l'explication qu'en donne Reumaux (1998:510): «Je lis les rouges-gorges, le Cravaté de Rouge (The One in Red Cravat). Aujourd'hui je sais ce qu'est cette cravate. Tout simplement le sens. Non pas la balle (la signification) que le poète envoie dans le langage mais l'extra-balle (l'instance paradoxale). La cravate du juge, celle du condamné. La cravate est la même. Les deux ont la corde au cou. Non pas le cravaté de rouge, mais l'Étranglé de Rouge». Sur les qualités morales que Dickinson attribue au merle, voir P. 250, 416, 685, 763, 864, 1059, 1542, 1919.

11. Voir, par exemple, le poème 1332, où Dickinson associe le merle à la fleur de mai ou épigée rampante (Mayflower ou Trailing arbutus); cette fleur sauvage, qui est l'emblème floral du Massachusetts (nommée d'après le bateau des Pilgrim Fathers), apparait, comme le merle, à la fonte des neiges. Dickinson associe également le merle à la marguerite, fleur des plus communes; Daisy était le surnom d'Emily.

12. Sur le mot «oblique», voir le poème 1129: Tell all the Truth but tell it slant.

13. Voir le poème 99 où Dickinson fait peut-être aussi allusion à ce phénomène climatique.

14. Dickinson distingue le sens générique de corn (céréale) et le sens américain d'Indian corn (maïs); wheat n'apparaît que dans le poème 1269 .

\section{RÉFÉRENCES}

Ansermoz-Dubois, Félix et Violette (1986) : Emily Dickinson. Poèmes, Lausanne, Éditions Ouverture.

Balzac, Honoré de (1949): «Les Paysans », La Comédie humaine, texte établi par Marcel Bouteron, Paris, Gallimard, «Bibliothèque de la Pléiade», t. VIII, p. 11-316.

Bosquet, Alain (1957): Emily Dickinson, Paris, Seghers.

Brooks, Van Wyck (1940): New England, Indian Summer, 1965-1915, New York, Dutton, p. 316329.

Denis, Philippe (1986) : «Emily Dickinson. Poèmes », La Revue de Belles-Lettres, 109-1-2, p. 6-21.

- (1986): Emily Dickinson. Quarante-sept poèmes, Genève, La Dogona.

_ (1988) : «Dix poèmes », La Nouvelle Revue Française, 421, p. 119-122.

Dickinson, Emily (1955): Poems, ed. by Thomas H. Johnson, Cambridge (Mass.), The Belknap Press of Harvard University Press, 3 vol.

- (1958) : Letters, ed. by Thomas H. Johnson and Theodora Ward, Cambridge (Mass.), The Belknap Press of Harvard University Press, 3 vol.

Forgue, Guy Jean (1970): Emily Dickinson. Poèmes, Paris, Aubier-Flammarion.

Frye, Northrop (1963): Fables of Identity, Studies in Poetic Mythology, New York and London, Harcourt Brace Jovanovich, p. 193-217.

Godfrey, W. Earl (1967): Les oiseaux du Canada, Ottawa, Musée national du Canada.

Leyris, Pierre (1995): Esquisse d'une anthologie de la poésie américaine du XIX ${ }^{e}$ siècle, Paris, Gallimard.

Malroux, Claire (1989): Emily Dickinson. Poèmes, Paris, Belin, «L'Extrême contemporain ».

— (1998): Emily Dickinson. Une âme en incandescence (poèmes), Paris, José Corti, coll. «Domaine romantique ».

_ (1999): Emily Dickinson. Lettres au maître, à l'ami, au précepteur, à l'amant, Paris, José Corti, coll. «Domaine romantique». 
Messian, Pierre (1956): Emily Dickinson, Poèmes choisis, Paris, Aubier-Montaigne.

Morency, Pierre (1992): Lumière des oiseaux, Montréal/Paris, Boréal/Seuil.

Niering, William A. et Nancy C. Olmstead (1979): The Audubon Society Field Guide to North American Wildflowers, New York/Toronto, Knopf/Random House.

Normand, Simone et Marcelle Fonfreide (1996): Emily Dickinson. 56 Poèmes, Paris, Le Nouveau Commerce.

Porte, Alain (1998) : «Emily Dickinson. 17 Poèmes», La Polygraphe, 1, p. 115-133.

Reumaux, Patrick (1990): Emily Dickinson: autoportrait au roitelet. Lettres à T.W Higginson et aux sours Norcross, Paris, Hatier, coll. "Terre étrangère ".

- (1998): Emily Dickinson. Le Paradis est au choix, Rouen, Librairie Élisabeth Brunet.

Scotт, Shirley L., dir. (1988): Guide d'identification des oiseaux de l'Amérique du Nord, National Geographic Society, trad. par M. Darveau, N. David, G. Falardeau, C. Simard et R. Simard, La Prairie, Éditions Broquet.

Sewall, Richard B. (1974): The Life of Emily Dickinson, New York, Farrar, Straus and Giroux.

TARI, Georges (1991): Emily Dickinson. Lettre au monde, [Valence], Editions du limon, coll. «L'arbre voyageur». 